\title{
Promoção à saúde do homem e os meios de comunicação como ferramenta na perspectiva do autocuidado
}

\author{
Promotion of men's health and the media as a tool from the perspective of self-care \\ Promoción de la salud masculina y los medios de comunicación como herramienta desde la \\ perspectiva del autocuidado
}

Recebido: 04/24/2021 | Revisado: 05/03/2021 | Aceito: 05/04/2021 | Publicado: 05/18/2021

Elizabeth Rose Costa Martins

ORCID: https://orcid.org/0000-0001-5947-5535

Universidade do Estado do Rio de Janeiro, Brasil E-mail: oigresrose@gmail.com

Karoline Lacerda de Oliveira

ORCID: https://orcid.org/0000-0001-8755-5858 Universidade do Estado do Rio de Janeiro, Brasil E-mail: lacerdakarol@hotmail.com

Andressa da Silva Medeiros

ORCID: https://orcid.org/0000-0001-8677-2543

Universidade do Estado do Rio de Janeiro, Brasil

E-mail: andressamedeirosmedeiros2@gmail.com

Gabriele Malta da Costa

ORCID: https://orcid.org/0000-0003-2780-0916

Universidade do Estado do Rio de Janeiro, Brasil

E-mail: gabrielemalta2005@hotmail.com

Letícia Guimarães Fassarella

ORCID: https://orcid.org/0000-0002-3903-7383

Universidade do Estado do Rio de Janeiro, Brasil

E-mail: lelefassarella@gmail.com

Nizélia Ferreira da Silva Floro Rosa

ORCID: https://orcid.org/0000-0003-2841-0848

Universidade do Estado do Rio de Janeiro, Brasil

E-mail:nizelia.floro@gmail.com

Samara de Andrade Ferreira

ORCID: https://orcid.org/0000-0002-3961-3213

Universidade do Estado do Rio de Janeiro, Brasil

E-mail: samara.ferreira.a18@gmail.com

Jady Assis de Souza

ORCID: https://orcid.org/0000-0003-2918-9209

Universidade do Estado do Rio de Janeiro, Brasil

E-mail: jadysouza@hotmail.com

Elisa da Conceição Silva Barros

ORCID: https://orcid.org/0000-0002-4279-9450

Universidade do Estado do Rio de Janeiro, Brasil E-mail: elisabarrosrb@gmail.com

Henrique Francisco de Sena

ORCID: https://orcid.org/0000-0003-0146-298X Universidade do Estado do Rio de Janeiro, Brasil E-mail: Henrique.francisco.sena@hotmail.com

Fabiana Cristina Silva da Rocha

ORCID: https://orcid.org/0000-0001-7665-0550

Universidade do Estado do Rio de Janeiro, Brasil

E-mail: fabianacsrocha@hotmail.com

\begin{abstract}
Resumo
Objetivos: Descrever a percepção do homem sobre a importância dos meios de comunicação para a sua saúde e discutir o impacto dos meios de comunicação como ferramenta, na promoção da saúde do homem voltado ao autocuidado. Metodologia: Estudo descritivo e exploratório, com abordagem qualitativa, desenvolvido com 27 homens a partir de 18 anos, devidamente matriculados numa universidade pública, situada no município do Rio de Janeiro. O instrumento de coleta de dados foi a entrevista semiestruturada e, para análise dos dados, foi aplicada a técnica de análise de conteúdo, conforme etapas: pré-análise, exploração do material e tratamento dos resultados, inferência e interpretação. Resultados e Discussão: O estudo evidenciou a percepção do homem sobre os meios de
\end{abstract}


comunicação e as informações para a sua saúde e a invisibilidade das ações de promoção à saúde sobre a população masculina nas mídias digitais. A análise apontou que os homens do estudo não se percebem contemplados pelos meios de comunicação, quanto à temática saúde, e observam campanhas voltadas para a população feminina e infantil, sendo a exceção o "Novembro Azul" como campanha dedicada à população masculina. Compreendem, ainda, a importância dos meios de comunicação como ferramenta para estímulo, esclarecimentos e reflexão sobre assuntos que envolvem as questões do gênero masculino em relação à saúde e ao autocuidado. Considerações finais: Diante da invisibilidade das ações de promoção à saúde sobre a população masculina nas mídias digitais, são necessárias campanhas de fácil entendimento, com a compreensão de integralidade em saúde, sob a perspectiva relacional de gênero.

Palavras-chave: Saúde do homem; Meios de comunicação; Autocuidado; Enfermagem.

\begin{abstract}
Objectives: To describe man's perception of the importance of the media for his health and to discuss the impact of the media as a tool in the promotion of man's health aimed at self-care. Methodology: Descriptive and exploratory study, with a qualitative approach, developed with 27 men from 18 years old, duly enrolled in a public university, located in the municipality of Rio de Janeiro. The data collection instrument was the semi-structured interview and, for data analysis, the content analysis technique was applied, according to the stages: pre-analysis, exploration of the material and treatment of the results, inference and interpretation. Results and Discussion: The study showed the perception of men about the means of communication and information for their health and the invisibility of health promotion actions on the male population in digital media. The analysis pointed out that the men in the study do not perceive themselves as contemplated by the media, regarding the health theme, and observe campaigns aimed at the female and child population, with the exception of "Novembro Azul" as a campaign dedicated to the male population. They also understand the importance of the media as a tool for stimulating, clarifying and reflecting on issues involving male issues in relation to health and self-care. Final considerations: In view of the invisibility of health promotion actions on the male population in digital media, easy-to-understand campaigns are needed, with an understanding of integrality in health, from a gender perspective.
\end{abstract}

Keywords: Men's health; Media; Self-care; Nursing.

\title{
Resumen
}

Objetivos: Describir la percepción del hombre sobre la importancia de los medios de comunicación para su salud y discutir el impacto de los medios de comunicación como herramienta en la promoción de la salud del hombre orientada al autocuidado. Metodología: Estudio descriptivo y exploratorio, con abordaje cualitativo, desarrollado con 27 hombres mayores de 18 años, debidamente matriculados en una universidad pública, ubicada en el municipio de Rio de Janeiro. El instrumento de recolección de datos fue la entrevista semiestructurada y, para el análisis de los datos, se aplicó la técnica de análisis de contenido, según las etapas: preanálisis, exploración del material y tratamiento de los resultados, inferencia e interpretación. Resultados y Discusión: El estudio mostró la percepción de los hombres sobre los medios de comunicación e información para su salud y la invisibilidad de las acciones de promoción de la salud de la población masculina en los medios digitales. El análisis señaló que los hombres del estudio no se perciben a sí mismos como contemplados por los medios, en cuanto al tema de la salud, y observan campañas dirigidas a la población femenina e infantil, a excepción de "Novembro Azul" como campaña dedicada a la población masculina. También comprenden la importancia de los medios de comunicación como herramienta para estimular, esclarecer y reflexionar sobre temas que involucran temas masculinos en relación con la salud y el autocuidado. Consideraciones finales: Ante la invisibilidad de las acciones de promoción de la salud en la población masculina en los medios digitales, se requieren campañas de fácil comprensión, con una comprensión de la integralidad en salud, desde una perspectiva de género.

Palabras clave: Salud de los hombres; Medios de comunicación; Autocuidado; Enfermería.

\section{Introdução}

A presença masculina nos espaços de saúde vem sendo um desafio para a saúde do homem. As campanhas voltadas para essa população estão sempre vinculadas ao câncer de próstata; além disso, a percepção é a de que o homem não procura os serviços de saúde, dando-lhes uma invisibilidade que acaba por ter, como consequência, a ausência da população masculina, no que diz respeito aos cuidados preventivos.

Dá-se, então, a reivindicação de uma política mais integral voltada à saúde masculina, pois, assim como as mulheres, os homens devem ser vistos de acordo com sua singularidade para que haja uma aproximação entre eles e os serviços de saúde. Tal conhecimento sobre as suas singularidades possibilita uma melhor aproximação do homem nos serviços de saúde, pois assim ele se sente pertencente e acolhido por esse programa (Gomes et al., 2011). 
Em 2008, surge então a Política Nacional de Atenção Integral à Saúde do Homem (PNAISH), cujo objetivo geral é o de:

promover a melhoria das condições de saúde da população masculina do Brasil, contribuindo, de modo efetivo, para a redução da morbidade e mortalidade através do enfrentamento racional dos fatores de risco e mediante a facilitação ao acesso, às ações e aos serviços de assistência integral à saúde (Brasil, 2008).

Mesmo com a criação da PNAISH, muito pouco ainda se conhece sobre o seu conteúdo e seus objetivos, pois não há uma divulgação consolidada dessa política e nem a sua implantação nos serviços de saúde para que haja atenção efetiva da população masculina no serviço primário de saúde, na promoção de sua saúde e autocuidado.

Segundo o Ministério da Saúde, no Brasil, os homens vivem, em média, 7,1 anos a menos do que as mulheres e, segundo o Instituto Brasileiro de Geografia e Estatística (IBGE), a perspectiva de vida da população masculina chegou a 72,2 anos, enquanto a feminina atingiu 79,3. Percebe-se que esse desconhecimento sobre a saúde do homem e a falta de informação acaba tendo uma repercussão e um impacto negativos sobre os índices de morbimortalidade masculina, assim como sua expectativa de vida (Brasil, 2018).

Um dos dados bem alarmantes está relacionado a doenças infecciosas e parasitárias onde o HIV representou a quinta causa de mortalidade no ano de 2014, com um total de 8.1562 óbitos, onde $64 \%$ desses óbitos ocorreram em homens. Tornando assim um desafio aos serviços de saúde, para que utilizem dos meios de comunicação como ferramenta para a mudança desses dados e desse quadro, que vem aumentando constantemente (Brasil, 2018).

Dessa forma, alguns autores, como Gomes et al. (2011), vêm chamando a atenção para o despertar das ações de promoção à saúde a fim de que a população masculina seja orientada quanto à realização precoce de exames. Assim, a implementação de programas e ações que busquem intensificar as estratégias de promoção à saúde e prevenção de agravos, bem como a redução da morbimortalidade, poderá trazer contribuições para o controle de doenças.

A comunicação é uma ferramenta indispensável para o entendimento, entrosamento e familiarização sobre diversos temas no que diz respeito à divulgação de um determinado assunto e pode ser verbal ou não verbal. A verbal está relacionada ao uso da linguagem falada e escrita, enquanto a não verbal se refere a posturas, gestos, expressões faciais, tom de voz - entre outros (Moreira, Nobrega \& Silva, 2003).

A comunicação em saúde é fundamental para melhorar o conhecimento do homem sobre si mesmo e precisa ter uma linguagem clara e informações suficientes para que possa ser compreendida pelo paciente e ele possa adquirir determinadas atitudes para promover a sua saúde, como realizar medidas preventivas e buscar por um serviço quando necessário (Moreira et al., 2003).

Em uma era de tecnologia, na qual as notícias surgem e se espalham em uma velocidade extremamente rápida, os serviços de saúde estão se adaptando cada vez mais a esse momento para que mais pessoas sejam alcançadas. Folhetos, materiais educativos, internet, twitter, entre outros, são utilizados para a propagação de mensagens, campanhas e conteúdo nos meios de comunicação (Lima et al., 2014). Apesar de os meios de comunicação estarem extremamente presentes, não há campanhas, além do "Novembro Azul", que, de fato, chamem o público masculino para cultivar o hábito de promoverem o seu autocuidado sem que se sintam tão femininos. Vê-se então a importância - e ao mesmo tempo o desafio - de os serviços de saúde utilizarem os meios de comunicação de forma a incentivar a presença dos homens na unidade, a fim de cuidarem da sua saúde.

Um dos princípios do Sistema Único de Saúde (SUS) é a equidade, que significa atender os indivíduos de acordo com as suas necessidades; porém, para que se chegue a esse princípio, a fim de acolher os indivíduos nos serviços de saúde, é 
necessário combater uma grande assimetria existente entre mulher e homem, fortalecendo a desvantagem entre gêneros presente durante o acolhimento (Gomes, 2003).

O autocuidado é realizado pelo indivíduo com o objetivo de promover a sua saúde, podendo ser de forma preventiva, como tratamento ou de modo a evitar um agravo, por exemplo. Entretanto, essas medidas devem ser ensinadas para o indivíduo. O profissional de saúde deve guiá-lo para que ele possa entender cada passo do procedimento, sua importância e suas implicações, bem como deve ensiná-lo a identificar alterações na saúde (Rub et al., 2019).

Essas medidas são fundamentais, pois há um afastamento do homem relativo a medidas de autocuidado e isso é consequência de alguns fatores, como a visão que a sociedade impõe sobre o que é ser homem - um ser forte e que não adoece, um ser invulnerável. Isso afeta a figura masculina, impossibilitando os homens, às vezes, de assumirem que o seu corpo apresenta alguma alteração e, consequentemente, de buscarem um atendimento imediato, o que geralmente os leva a solicitarem ajuda quando o caso se tornou mais grave. Outro fator é o desconhecimento sobre as possíveis doenças que o seu corpo pode adquirir, seus sinais e as medidas preventivas que podem realizar (Botton, Cunico, \& Strey, 2017).

As autoras, durante sua trajetória como acadêmicas de enfermagem, com participação na Iniciação Científica e no Projeto de Extensão sobre a Saúde do Homem, compreendem a importância do cuidado da saúde na perspectiva do gênero masculino. $\mathrm{O}$ interesse pela temática surgiu com o intuito de investigar o impacto dos meios de comunicação como ferramenta na perspectiva do autocuidado em saúde desta população.

O estudo tem como objeto a promoção à saúde do homem na perspectiva do autocuidado tendo os meios de comunicação como ferramenta.

Visando elucidar o objeto de estudo, foram delimitados os seguintes objetivos: Descrever a percepção do homem sobre a importância dos meios de comunicação para a sua saúde e discutir o impacto dos meios de comunicação como ferramenta, na promoção da saúde do homem voltado ao autocuidado.

\section{Metodologia}

Estudo descritivo de abordagem qualitativa, realizado em uma universidade pública, situada no município do Rio de Janeiro.

A fim de atender ao objeto de estudo, buscou-se criar alguns critérios para seus participantes, como a faixa etária. Nesse sentido, foi definida a faixa etária dos 18 aos 59 anos, a partir do recorte metodológico estratégico estabelecido pela PNAISH, que engloba a parcela preponderante de força produtiva e representa um significativo papel sociocultural e político, correspondente a $41,3 \%$ da população (Brasil, 2008).

Como critérios de inclusão foram selecionados: os homens a partir de 18 anos, devidamente matriculados na grade universitária, presentes no momento da coleta de dados, sem restrições para o curso, horário ou período acadêmico. O critério de exclusão foi o de homens que se utilizam do espaço da universidade como rota alternativa, sem um vínculo com a instituição; dessa forma, o estudo contou com 27 participantes.

A pesquisa foi realizada no período de dezembro de 2019 a fevereiro de 2020, após aprovação do Comitê de Ética e Pesquisa sob o $\mathrm{n}^{\mathrm{o}}$ 4.457.874. O estudo seguiu os aspectos éticos e as determinações contidas na Resolução 466/2012 do Conselho Nacional de Saúde.

Como instrumento de coleta de dados, foi utilizada a entrevista semiestruturada, que parte de certos questionamentos básicos apoiados em teorias que interessam à pesquisa e que, em seguida, oferecem amplo campo de interrogativas (Minayo, 2013). 
A entrevista foi composta de duas partes: a primeira, caracterizando os participantes segundo a faixa etária, identidade de gênero, estado civil, curso de graduação; a segunda, com questões abertas sobre a promoção da saúde na perspectiva de gênero e os meios de comunicação como facilitadores ao autocuidado.

Vale ressaltar que foram realizadas 27 entrevistas, conforme a disponibilidade dos discentes, nas dependências da universidade, situada no município do Rio de Janeiro. Elas ocorreram de forma individualizada, em um espaço apropriado para a sua realização, sem comprometer seu desenvolvimento.

Cada participante recebeu um termo de consentimento livre e esclarecido (TCLE), no qual todos foram orientados quanto aos objetivos do estudo, a relevância, a metodologia, assim como a garantia da participação voluntária, do sigilo e do anonimato, da possibilidade de desistência da participação na pesquisa e dos possíveis riscos mínimos relacionados à pesquisa.

Com o objetivo de resguardar a identidade dos participantes, foram utilizados códigos de identificação, optando-se pela escolha da letra $\mathrm{H}$, seguida por um número ordinal identificando a ordem das entrevistas realizadas. As entrevistas foram gravadas em formato de áudio, por meio de um aparelho eletrônico, para garantia da fidedignidade das informações coletadas $\mathrm{e}$, posteriormente, transcritas.

Após a coleta, os dados foram transcritos e organizados. Com a intenção de conhecer os significados por trás dos discursos emanados durante as entrevistas, o método utilizado foi o da análise de conteúdo, pois tudo que é dito é susceptível de ser submetido a este tipo de análise (Bardin, 2016).

O objeto da análise de conteúdo é a palavra, aspecto individual da linguagem; seu objetivo são os significados dos vocábulos, tentando compreender os atores e o ambiente onde estão inseridos; é organizada em três etapas: pré-análise; exploração do material e tratamento dos resultados; inferência e interpretação (Bardin, 2016).

Para a sistematização dos achados, realizou-se leitura flutuante, a qual fez emergir 198 unidades de registro, organizadas em duas categorias: Percepção do homem sobre os meios de comunicação e as informações para a sua saúde e A invisibilidade das ações de promoção à saúde sobre a população masculina nas mídias digitais.

\section{Resultados e Discussão}

Os participantes do estudo tinham a seguinte caracterização: em relação à faixa etária, destaca-se a predominância de 20 a 25 anos, de 22 (81\%) participantes; de 26 a 30 anos, de 4 (15\%) e acima de 30 anos, 1 (4\%) participante. Quanto ao estado civil, todos eram solteiros, sendo que 15 (56\%) eram graduandos da área de ciências biológicas e 12 (44\%) eram estudantes de ciências exatas. No que refere à identidade de gênero, houve predomínio daqueles que se identificaram com o gênero masculino.

\subsection{Percepção do homem sobre os meios de comunicação e as informações para a sua saúde}

A fim de compreender a percepção do homem sobre a importância dos meios de comunicação para a sua saúde, na perspectiva do autocuidado, podem-se perceber as dificuldades, conforme as falas:

Homens não têm muito costume de procurar informações e nem de se cuidar. (H.5)

Acho que, em comparação à população feminina, é muito menor; a mulher se preocupa muito mais com a saúde, porque os homens não costumam procurar informações (H.1) 
A resistência dos homens em buscar os serviços de saúde, associada à falta de prevenção e autocuidado e à fragilidade e insegurança, contrasta com sua virilidade, sua exposição a situações de risco e sentimento de invulnerabilidade, expressando traços culturais de uma visão hegemônica de masculinidade (Schwarz, 2012).

Nesse sentido, percebe-se a dificuldade dos homens na busca de informação que envolva a sua saúde, surgindo a questão de que o cuidar é feminino; por isso, quem procura as informações são as mulheres. De alguma forma, esse sentimento é fortalecido pelos meios de comunicação, nos quais se percebe maior destaque às questões do gênero feminino.

Precisa melhorar a linguagem utilizada nas informações. (H.19)

É preciso abordar a temática de uma maneira mais descontraída, e que nos diz respeito. (H.13)

Se não for simples, o homem não tem predisposição para ouvir e desiste. (H.3)

Percebo a necessidade de informações que interessam à população masculina. (H.22)

Segundo Almeida (2012), para a compreensão das informações, deve ser utilizada uma linguagem clara e de fácil compreensão para a população. Vale ressaltar a importância de trazer informações de interesse da população masculina, atendendo às suas particularidades de forma compreensiva e fidedigna.

De acordo com Monari e Bertolli Filho (2019), as fakes news têm se tornado cada vez mais fortes dentro de diversos segmentos e, sobretudo, na saúde. Sendo assim, interfere no cuidado de seus usuários, uma vez que essas informações inverídicas são facilmente propagadas, tendo em vista a linguagem e solução simples e ilusória, reforçando ainda mais a necessidade de campanhas e outras iniciativas para o combate das falsas informações. Essa deficiência é confirmada nas seguintes falas:

Hoje em dia existe muita disseminação de fake news, então é muito fácil ser enganado. (H.19)

É preciso buscar conhecimento em fontes confiáveis. (H.4)

Se as fontes não forem sérias, poderá prejudicar minha saúde. (H.10)

Há ainda uma dificuldade de compreender que o homem é o principal sujeito responsável pelo seu cuidado e manutenção de sua saúde; em contrapartida, se não há meios digitais que reforcem essa prática, torna-se ainda mais difícil a construção dessa visão entre a população masculina (Martins et al., 2020a).

Os participantes reconhecem que existe um déficit no autocuidado na perspectiva do gênero masculino e reforçam a importância das mídias na prevenção de doenças, conforme as falas:

É importante, pois estatisticamente os homens morrem antes das mulheres e têm uma saúde pior, pela falta de autocuidado. (H.12)

Principalmente pela conscientização, seguido do estímulo em procurar não só mais informações, como também a incentivar os homens a procurar maiores cuidados com sua própria saúde. (H.3)

Relembrando o homem que sua saúde é importante de ser cuidada, ainda que, muitas vezes, esse cuidado seja taxado de forma negativa e debochada. (H.6) 
Além da ausência do autocuidado, predomina a visão da virilidade nessa população, o que reforça a falta de adesão às práticas de saúde. Como trazem Martins et al. (2020b), esta situação, de alguma forma, fortalece a importância dos meios de comunicação em trazer temas de interesse, que reforcem a promoção à saúde e prevenção de doenças à população masculina.

Acredito que ainda exista o pensamento de 'sou homem, sou forte, não preciso de médico', algo que me envergonha um pouco. É preciso tratar a saúde como algo sério, não só a física como a psicológica; apenas quando todos entenderem que problemas não são frescuras, as coisas melhorarão. (H.22)

Acho que o problema não é só a falta de informações, mas a falta de consenso em torno de 'homens deviam buscar se cuidar'. (H.11)

Para a saúde do homem, acredito que o principal seja conseguir derrubar esta barreira que o homem tem que ser forte e não pode reclamar de dor ou doença. (H.15)

Rocha et al. (2020) afirmam que o machismo é um dos principais fatores que contribuem para a dificuldade do homem de se perceber vulnerável e buscar informações acerca da sua saúde.

Faz-se necessário que os meios de comunicação, de alguma forma, incentivem a população masculina a procurarem os serviços de saúde, trazendo informações que atendam às suas expectativas e necessidades, envolvendo o autocuidado.

Hoje, as informações a respeito do cuidado com a saúde do homem são limitadas, enquanto deveriam chamar sua atenção para a promoção da saúde e prevenção de doenças e agravos, deixando-o à vontade e acolhido, de modo a assegurar seu espaço e suas condições de gênero. As redes sociais podem ser uma das ferramentas para maior participação do gênero masculino, levando-o a refletir sobre seu papel em cuidar de sua saúde.

\subsection{A invisibilidade das ações de promoção à saúde sobre a população masculina nas mídias digitais}

As ações que envolvem a saúde da população masculina nas mídias sociais estão sempre voltadas ao câncer de próstata; no entanto, não se pode reduzir este homem a apenas uma doença, diante do fato de que o índice de morbimortalidade envolve outras doenças, como a cardiovascular, dentre outras.

Não divulgam muito sobre o cuidado com a saúde masculina por causa de conceitos já pré-estabelecidos, a única campanha amplamente divulgada é a do Novembro Azul. (H.3)

Tirando o Novembro Azul, que é a campanha pela prevenção ao câncer de próstata e o cuidado com a saúde, não é visto o ano inteiro nada relacionado ao autocuidado dos homens. (H.7)

Nesta nossa sociedade, não se tem este olhar mais específico para saúde do homem. (H.12)

Vale ressaltar a importância da construção de uma abordagem que inclua a promoção à saúde e à prevenção de agravos como ferramenta de comunicação, considerando a população masculina como protagonista de sua própria saúde.

Algumas falas reafirmam o déficit dos meios de comunicação, voltado à saúde na perspectiva do gênero masculino, mas apontam o incentivo ao consumo de produtos de beleza. 
Temos agora a campanha da Gillete com uma propaganda chamada 'The best men can be", que repudiava a masculinidade tóxica como assédio, bullying e tinham também como objetivo incentivar melhores relações entre homens. (H.11)

Clear Man, controle de caspa. (H.9)

Campanhas de desodorante, propagandas de roupas. (H.4)

Fontes, Borelli e Casotti (2012) trazem a insatisfação que existe na população masculina em ter sua imagem atrelada somente a elementos de beleza. Nesse sentido, a aparição na mídia apresenta a visão de um homem metrossexual, que se importa somente com a sua saúde física e corporal. Com isso, não se observam campanhas chamativas a esta população, que reforcem a importância do bem-estar no âmbito da promoção à saúde e, principalmente, na prevenção de agravos.

A mídia tem um papel fundamental de veiculação de informação; então, se divulgasse mais coisas relacionadas à prevenção da saúde do homem, mais homens teriam a consciência que isso deve ser feito. (H.10)

Tendo o homem um papel social de provedor da família, a mídia projeta nele uma visão capitalista, por ser a figura que sustenta financeiramente seus dependentes. Sendo assim, é mais vantajoso oferecer produtos para consumo que o leve por instinto a comprar e gerar lucro.

Como nossa sociedade segue o regime capitalista, é observada a questão do conflito de interesse. Afinal, dentro da divulgação de uma informação para saúde pode estar a indução e tentativa de influenciar para o consumo de determinados produtos, serviços, formas de se fazer saúde e de ideias. (H.17)

Percebe-se a invisibilidade de ações de promoção à saúde na perspectiva do gênero masculino, pelos meios de comunicação. Os recursos como propagandas, informativos, mídias digitais, dentre outros, são escassos e, de alguma forma, não atendem às particularidades e necessidades desta população.

\section{Considerações Finais}

A invisibilidade masculina é notória no que tangencia campanhas voltadas para a sua saúde, destacando-se apenas o câncer de próstata. Tal fato reflete uma baixa adesão de homens aos serviços de Atenção Primária à Saúde e cuidados preventivos. Diante disso, origina-se a Política de Atenção Integral à Saúde do Homem, com o intuito de melhorar a perspectiva da saúde masculina no Brasil, porém a política ainda não é amplamente divulgada, sendo pouco conhecida pela população.

Ressalta-se que comunicação em saúde, seja ela verbal ou não verbal, é uma estratégia fundamental para familiarizar a população masculina com sua saúde, trazendo o autoconhecimento sobre o corpo, mostrando suas vulnerabilidades e necessidades em saúde, minimizando, assim, os impactos advindos da falta de conhecimento, prevenção e autocuidado.

Os homens do estudo não se percebem contemplados pelos meios de comunicação, quanto à temática saúde, pois observam, sobretudo, campanhas voltadas para as populações feminina e infantil, sendo a exceção o "Novembro Azul" câncer de próstata, como campanha dedicada à população masculina. 
Apontam, ainda, a necessidade da difusão de conhecimentos voltados à promoção da saúde e à prevenção de doenças, pois sabe-se que os homens têm um déficit de autocuidado e esta situação é expressa pelos índices de morbimortalidade mais elevados entre estes indivíduos.

Sendo assim, os homens compreendem a importância dos meios de comunicação como ferramenta para estímulo, esclarecimentos e reflexão sobre assuntos que envolvem as questões do gênero masculino. Com isto, reforça-se a necessidade de haver campanhas de fácil entendimento, com maior divulgação e voltadas à população masculina. No que envolve a promoção à saúde e a prevenção de agravos, voltadas à saúde do homem, existe um desafio a ser enfrentado, que é o acesso a informações que estimule este indivíduo a pensar em cuidar de sua saúde, pois a falta de visibilidade dessa população traz consequências que se traduzem no aumento de sua morbimortalidade.

A enfermagem deve participar de ações, tendo os meios de comunicação como ferramenta, através das mídias digitais, incentivando as práticas de autocuidado, relacionadas às particularidades dessa população, na perspectiva do gênero.

O estudo apresenta limitações ao retratar a realidade de alguns homens de uma universidade pública e, dessa forma, os resultados não podem ser generalizados para a população masculina de todas as universidades, apesar de os resultados condizerem com demais investigações nacionais.

\section{Referências}

Almeida, M. A. (2012). A promoção da saúde nas mídias sociais: uma análise do perfil do Ministério da Saúde no Twitter. Faculdade de Comunicação e Biblioteconomia. Universidade Federal de Goiás, Goiânia. http://repositorio.bc.ufg.br/bitstream/ri/4480/5/TCCE\%20\%20Mar\%C3\%ADlia\%20de\%20Almeida\%20e\%20Almeida\%20-\%202012.

Bardin, L. (2016). Análise de conteúdo. Tradução Luís Antero Reto, Augusto Pinheiro: Edições 70.

Botton, A., Cunico, S. D., \& Strey, M. N. (2017). Diferença de gêneros no acesso aos serviços de saúde: problematizações necessárias. Mudanças- Psicologia da Saúde. 25(21), 67-72, jan./jun. https://doi.org/10.15603/2176-1019/mud.v25n1p67-72.

Fontes, O. A., Borelli, F. C., \& Casotti, L. M. (2012). Como ser homem e ser belo? Um estudo exploratório sobre a relação entre masculinidade e o consumo de beleza. REAd. Rev. eletrôn. adm. 18(2), 400-432. https://doi.org/10.1590/S1413-23112012000200005

Gomes, R., Moreira, M. C. N., Nascimento, E. F., Rebello, L. E. F. S., Couto, M. T., \& Schraiber, L. B. (2011). Os homens não vêm! Ausência e/ou invisibilidade masculina na atenção primária. Cad Saúde Pública. 16(supl. 1), 983-92. http://dx.doi.org/10.1590/S1413-81232011000700030

Gomes, R. (2003) Sexualidade masculina e saúde do homem: proposta para uma discussão. Cien Saúde Colet. 8(3), 825-9. https://doi.org/10.1590/S141381232003000300017.

Lima, B., Martins, E. R. C., Ramos, R. C. A., Cristiane Maria Amorim Costa, C. M. A., Clos, A. C., \& Ferreira, J. I. C. (2014). Os meios alternativos de comunicação como ferramenta para a prevenção do câncer de próstata. Rev. enferm UERJ. Rio de Janeiro, $22(5)$, 656-62. http://dx.doi.org/10.12957/reuerj.2014.15669.

Martins, E. R. C., et al. (2020a). Saúde do homem jovem e as práticas educativas na perspectiva da promoção a saúde. Research, Society and Development. 9(9), e400997351. http://dx.doi.org/10.33448/rsd-v9i9.7351

Martins, E. R. C., et al. (2020b). Ações extensionistas para a saúde do homem: um relato de experiência. Research Society and Development. 9(11), e1579119687. http://doi.org/10.33448/rsd-v9i11.9687.

Ministério da Saúde. (2008). Política Nacional de Atenção Integral à Saúde do Homem: Plano de Ação Nacional. http://portalsaude.saude.gov.br/i ages/pdf/2014/maio/21/CNSH-DOC-Plano-Nacional.PNAISH-2009-2011.pdf.

Ministério da Saúde. Secretaria de Atenção à Saúde. Departamento de Ações Programáticas Estratégicas. (2018). Perfil da morbimortalidade masculina no Brasil [recurso eletrônico] / Ministério da Saúde, Secretaria de Atenção à Saúde, Departamento de Ações Programáticas Estratégicas: Ministério da Saúde. ISBN 978-85-334-2575-0 1 .

Minayo, M. C. S. (2013). O desafio do conhecimento: pesquisa qualitativa em saúde: Hucitec.

Monari, A. C. P., \& Bertolli, F. C. (2019). Saúde sem fake news: Estudo e caracterização das informações falsas divulgadas no canal de informação e checagem de fake news do Ministério da Saúde. Revista Mídia e Cotidiano. 13(1), 160-186. https://pdfs.semanticscholar.org/f875/dec4fde098fb73081b7f8516d9c8508705a2.pdf.

Moreira, M. F., Nobrega, M. M. L., \& Silva, M. I. T. (2003). Comunicação escrita: contribuições para a elaboração de material educativo em saúde. Rev. Bras. Enf. 56(2), 184-8. https://doi.org/10.1590/S0034-71672003000200015.

Rocha, F C. S., et al. (2020). Acolhimento da população masculina sob a percepção dos profissionais de enfermagem: desconstrução da invisibilidade. Research Society and Development. 9(12) e6791210708. http://doi.org/10.33448/rsd-v9i12.10708. 
Research, Society and Development, v. 10, n. 6, e0410615421, 2021

(CC BY 4.0) | ISSN 2525-3409 | DOI: http://dx.doi.org/10.33448/rsd-v10i6.15421

Rub, M. B. C., Medrano, C., Silva, C. D., Wink, S., Liss, P. E., \& Santos, E. K. A. (2006). A noção de cuidado de si mesmo e o conceito de autocuidado na enfermagem. Texto Contexto Enferm. Florianópolis, 15(esp.), 152-7. http://www.scielo.br/pdf/tce/v15nspe/v15nspea18.

Schwarz, E. (2012). Reflexões sobre o gênero e a Política Nacional de Atenção Integral à Saúde do Homem. Ciência e Saúde Coletiva. 17(10), 2581-83. https://doi.org/10.1590/S1413-81232012001000004 